PROCEEDINGS OF THE

AMERICAN MATHEMATICAL SOCIETY

Volume 132, Number 11, Pages 3305-3308

S 0002-9939(04)07474-X

Article electronically published on May 21, 2004

\title{
THE IDENTITY IS ISOLATED AMONG COMPOSITION OPERATORS
}

\author{
C-H. CHU, R. V. HÜGLI, AND M. MACKEY \\ (Communicated by Joseph A. Ball)
}

\begin{abstract}
Let $H^{\infty}(B)$ be the Banach algebra of bounded holomorphic functions on the open unit ball $B$ of a Banach space. We show that the identity operator is an isolated point in the space of composition operators on $H^{\infty}(B)$. This answers a conjecture of Aron, Galindo and Lindström.
\end{abstract}

\section{INTRODUCTION}

Let $B$ be the open unit ball of a complex Banach space $E$, and let $H^{\infty}(B)$ be the uniform algebra of bounded complex-valued holomorphic functions on $B$, with the supremum norm $\|f\|=\sup _{x \in B}|f(x)|$. Given any holomorphic self-map $\phi$ of $B$, we define the composition operator $C_{\phi}: H^{\infty}(B) \rightarrow H^{\infty}(B)$ by

$$
C_{\phi}(f)=f \circ \phi \quad\left(f \in H^{\infty}(B)\right) .
$$

The collection of such operators with the operator norm topology is denoted by $\mathcal{C}\left(H^{\infty}(B)\right)$. This space has been widely studied, and recently, Aron, Galindo and Lindström [1] have determined its path connected components for some special Banach spaces $E$, thereby extending results of MacCluer, Ohno and Zhao [6] for the case when $B$ is the open unit disc $\Delta$ in the complex plane. A main result in [1] is

Theorem 1.1 ([1, Theorem 16]). If $E=C_{0}(X)$ or $E$ is a Hilbert space, then the composition operators $C_{\phi}$ and $C_{\psi}$ lie in the same path connected component in $\mathcal{C}\left(H^{\infty}(B)\right)$ if and only if $\left\|C_{\phi}-C_{\psi}\right\|<2$.

Furthermore, using techniques involving w-strong peak points and determining sets for $H^{\infty}(B)$ when $B$ belongs to some special Banach spaces, the following result is established.

Theorem 1.2 ([1, Corollary 12]). The identity operator is an isolated point in $\mathcal{C}\left(H^{\infty}(B)\right)$ when $E$ is $C_{0}(X)$ or $\ell_{1}$ or any strictly convex reflexive Banach space.

Two open questions were raised in [1]. First, does Theorem 1.1 hold when $E$ is a $J B^{*}$-triple? A positive answer to this question has been given in [7]. The second conjecture is that Theorem 1.2 holds for every Banach space $E$. We give a positive

Received by the editors May 7, 2003 and, in revised form, August 12, 2003.

2000 Mathematics Subject Classification. Primary 47B38, 46J15, 46G20, 32A10.

Key words and phrases. Composition operator, holomorphic function on Banach space, Carathéodory distance. 
answer and a simple proof in this paper. We use only the hyperbolic metric, but do not require w-strong peak points.

\section{Proof of the conjecture}

The space of all complex-valued homomorphisms on $H^{\infty}(B)$ forms the maximal ideal space of $H^{\infty}(B)$ and contains, in particular, the point evaluation functionals $\left\{\delta_{x}: x \in B\right\}$. The pseudo-hyperbolic distance on the maximal ideal space is defined by

$$
\beta(m, n)=\sup \left\{|\hat{f}(n)|: f \in H^{\infty}(B),\|f\| \leq 1, \hat{f}(m)=0\right\}
$$

where $\hat{f}$ is the Gelfand transform of $f$. We note from [1, Remark 2] that

$$
\left\|C_{\phi}-C_{\psi}\right\|<2 \quad \text { if and only if } \quad \sup _{x \in B} \beta\left(\delta_{\phi(x)}, \delta_{\psi(x)}\right)<1 .
$$

The Carathéodory distance on $B$ is given by

$$
C_{B}(x, y)=\sup \{\gamma(f(x), f(y)): f \in H(B, \Delta)\}
$$

for $x, y \in B$, where $\gamma$ is the Poincaré metric on the disc $\Delta$ and $H(B, \Delta)$ the space of holomorphic maps from $B$ to $\Delta$. Both $C_{B}$ and $\beta$ are contracted by holomorphic functions and preserved by biholomorphic functions.

The metric

$$
d_{B}(x, y):=\sup \left\{|f(x)-f(y)|: f \in H^{\infty}(B),\|f\| \leq 1\right\} \quad(x, y \in B)
$$

and its relation to the Carathéodory distance $C_{B}$ are examined in [7] where it is shown that

$$
d_{B}(x, y)=\frac{2-2 \sqrt{1-\left(\tanh C_{B}(x, y)\right)^{2}}}{\tanh C_{B}(x, y)} \quad(x, y \in B) .
$$

We have $d_{B}(x, y) \geq d_{\Delta}(h(x), h(y))$ for any $h \in H(B, \Delta)$. Also,

$$
d_{B}(x, y) \leq 2 \sup \left\{|f(x)|: f \in H^{\infty}(B),\|f\| \leq 1, f(y)=0\right\} .
$$

To see this, it suffices to note that for any $f \in H^{\infty}(B)$ with $\|f\| \leq 1$, the function $f^{y}$ defined by $f^{y}(x)=\frac{1}{2}(f(x)-f(y))$ is also in the closed unit ball of $H^{\infty}(B)$.

Let $E^{*}$ be the dual of a complex Banach space $E$. We denote the unit spheres of $E$ and $E^{*}$ by $S(E)$ and $S\left(E^{*}\right)$ respectively. Given $x \in S(E)$, we denote the set of support functionals of $x$ by

$$
\operatorname{supp}(x)=\left\{f \in E^{*}:\|f\|=f(x)=1\right\} .
$$

Let $T: E \longrightarrow E$ be a bounded complex linear operator. We recall that the spatial numerical range of $T$ is defined by

$$
V(T)=\{f(T x): x \in S(E), f \in \operatorname{supp}(x)\}
$$

(cf. [3]). By [3, Theorem 3.9.4], the numerical radius $v(T)$ of $T$ is given by

$$
v(T)=\sup \{|\lambda|: \lambda \in V(T)\} .
$$

We also have, by [3, Theorem 1.4.1],

$$
\|T\| \geq v(T) \geq \frac{1}{e}\|T\| .
$$

We now prove the conjecture that subsumes Theorem 1.2.

Theorem 2.1. Let $E$ be a Banach space with open unit ball B. Then the identity operator is an isolated point in the space of composition operators on $H^{\infty}(B)$. 
Proof. Let $I: H^{\infty}(B) \longrightarrow H^{\infty}(B)$ be the identity operator, and suppose that $C_{\phi}$ is in the component of $I$ for some holomorphic self-map $\phi$ of $B$. We show that $\phi$ is the identity map on $B$. We have $\left\|C_{\phi}-I\right\|<2$ by [5] and $\sup _{x \in B} \beta\left(\delta_{\phi(x)}, \delta_{x}\right)<1$ by (1). Since

$$
\begin{aligned}
\beta\left(\delta_{\phi(x)}, \delta_{x}\right) & =\sup \left\{\left|\hat{f}\left(\delta_{\phi(x)}\right)\right|: f \in H^{\infty}(B),\|f\| \leq 1, \hat{f}\left(\delta_{x}\right)=0\right\} \\
& =\sup \left\{|f(\phi(x))|: f \in H^{\infty}(B),\|f\| \leq 1, f(x)=0\right\} \\
& \left.\geq \frac{1}{2} \sup \left\{|f(\phi(x))-f(x)|: f \in H^{\infty}(B),\|f\| \leq 1\right\} \quad \text { (by (3) }\right)
\end{aligned}
$$

we see that

$$
\sup _{x \in B} d_{B}(\phi(x), x)<2
$$

and hence (2) gives

$$
\sup _{x \in B} C_{B}(\phi(x), x)<\infty
$$

or that

$$
\sup _{x \in B, f \in H(B, \Delta)} \gamma(f(x), f(\phi(x)))<\infty .
$$

Let $\lambda \in S\left(E^{*}\right)$ be norm-attaining; that is, there exists $x_{\lambda} \in S(E)$ with $\lambda\left(x_{\lambda}\right)=1$. Define $\psi: \Delta \rightarrow \Delta$ by $\psi(\zeta)=\lambda\left(\phi\left(\zeta x_{\lambda}\right)\right)$. Then $\psi$ is holomorphic, and we have

$$
\begin{aligned}
\sup _{\zeta \in \Delta} \gamma(\zeta, \psi(\zeta)) & =\sup _{\zeta \in \Delta} \gamma\left(\lambda\left(\zeta x_{\lambda}\right), \lambda\left(\phi\left(\zeta x_{\lambda}\right)\right)\right) \\
& \leq \sup _{x \in B} \gamma(\lambda(x), \lambda(\phi(x))) \\
& \leq \sup _{x \in B, f \in H(B, \Delta)} \gamma(f(x), f(\phi(x)))<\infty .
\end{aligned}
$$

Since $\gamma(\zeta, \psi(\zeta))=\tanh ^{-1} \beta(\zeta, \psi(\zeta))$ on $\Delta$, we have $\sup _{\Delta} \beta(\zeta, \psi(\zeta))<1$ and it follows from the one-dimensional result that $\psi=i d_{\Delta}$. Hence we have

$$
\zeta=\lambda\left(\phi\left(\zeta x_{\lambda}\right)\right) \quad(\zeta \in \Delta)
$$

In particular, we have $\lambda(\varphi(0))=0$. By the Bishop-Phelps theorem [2], the normattaining functionals in $E^{*}$ are norm-dense in $E^{*}$. Therefore $\phi(0)=0$. Let us write (5) in the form

$$
i d_{\Delta}=\lambda \circ \phi \circ i_{x_{\lambda}}
$$

where $i_{x_{\lambda}}: \Delta \rightarrow B$ is the map $i_{x_{\lambda}}(\zeta)=\zeta x_{\lambda}$. Taking the derivative at $\zeta \in \Delta$ of both sides we obtain

$$
1=\lambda\left(\phi^{\prime}\left(\zeta x_{\lambda}\right)\left(x_{\lambda}\right)\right)
$$

which gives

$$
1=\lambda\left(\phi^{\prime}(0) x_{\lambda}\right) .
$$

The above arguments imply that, for any $x \in S(E)$ and $f \in \operatorname{supp}(x)$, we have $1=f\left(\phi^{\prime}(0) x\right)$. Let $T=\phi^{\prime}(0)-I$. We obtain

$$
\begin{aligned}
V(T) & =\{f(T x): x \in S(E), f \in \operatorname{supp}(x)\} \\
& =\left\{f\left(\phi^{\prime}(0) x\right)-f(x): x \in S(E), f \in \operatorname{supp}(x)\right\} \\
& =\{0\} .
\end{aligned}
$$

It follows from (4) that $\|T\|=0$. Hence $\phi^{\prime}(0)=I$. Since we have already established that $\phi(0)=0$, Cartan's uniqueness theorem asserts that $\phi$ itself is the identity map on $B$ as required (see [4, Proposition 6.6]). 
Corollary 2.2. Let $E$ be a Banach space and $\psi$ a biholomorphic self-map of the open unit ball $B$ of $E$. Then $C_{\psi}$ is isolated in $\mathcal{C}\left(H^{\infty}(B)\right)$.

Proof. The result is true for $\psi=i d$ from above. Now observe that $C_{\psi}$ is a homeomorphism of $\mathcal{C}\left(H^{\infty}(B)\right)$ that takes the identity to the composition operator $C_{\psi}$.

\section{ACKNOWLEDGEMENTS}

The second author wishes to thank the Department of Mathematics of University College Dublin for the hospitality received during his visit there, and the Swiss National Science Foundation for financial support. The third author gratefully acknowledges hospitality received at Queen Mary College, University of London.

\section{REFERENCES}

[1] R. Aron, P. Galindo And M. Lindström, Connected components in the space of composition operators, Integr. Equ. Oper. Theory, 45 (2003) 1-14. MR 2003m:47041

[2] E. Bishop And R. R. Phelps, A proof that every Banach space is subreflexive, Bull. Amer. Math. Soc., 67 (1961) 97-98. MR 23:A503

[3] F. F. Bonsall and J. Duncan, Numerical Ranges of Operators on Normed Spaces and of Elements of Normed Algebras, Vol. 2, London Math. Soc. Lect. Note Ser., Cambridge Univ. Press (1971) Cambridge. MR 44:5779

[4] S. Dineen, The Schwarz Lemma, Oxford Univ. Press (1989) Oxford. MR 91f:46064

[5] P. Galindo and M. Lindström, Factorization of homomorphisms through $H^{\infty}(D)$, J. Math. Anal. Appl. 280 (2003) 375-386.

[6] B. MacCluer, S. Ohno And R. Zhao, Topological structure of the space of composition operators on $H^{\infty}$, Integr. Equ. Oper. Theory, 40 (2001) 481-494. MR 2002d:47039

[7] M. Mackey And P. Mellon, A Schwarz lemma and composition operators, Integr. Equ. Oper. Theory (to appear).

School of Mathematical Sciences, Queen Mary College, University of London, LONDON E1 4NS, ENGLAND

E-mail address: c.chu@qmul.ac.uk

Department of Mathematics, University of California, Irvine, California 92697

E-mail address: remo.huegli@stat.unibe.ch

Department of Mathematics, University College, Dublin 4, Ireland

E-mail address: michael.mackey@ucd.ie 